\title{
Flavonoid components in Scutellaria baicalensis inhibit nicotine-induced proliferation, metastasis and lung cancer-associated inflammation in vitro
}

\author{
WEI-YI GONG，JIN-FENG WU，BAO-JUN LIU，HONG-YING ZHANG，YU-XUE CAO, \\ JING SUN, YU-BAO LV, XIAO WU and JING-CHENG DONG \\ Institute of Integrative Medicine, Department of Integrative Medicine, \\ Huashan Hospital, Fudan University, Shanghai 200041, P.R. China
}

Received December 25, 2013; Accepted February 12, 2014

DOI: $10.3892 /$ ijo. 2014.2320

\begin{abstract}
The objective of the present study was to investigate the therapeutic efficacy of flavonoid components in Scutellaria baicalensis on proliferation, metastasis and lung cancer-associated inflammation during nicotine induction in the A549 and H1299 lung cancer cell lines. After experimental period, augmentation of proliferation was observed, accompanied by marked decrease in apoptotic cells in nicotine-induced lung cancer cells; additionally, nicotine-exposed cells exhibited increased invasive and migratory abilities based on invasion and wound-healing assay. Flavones in Scutellaria, baicalin, baicalein and wogonin significantly counteracted the above deleterious changes. Moreover, assessment of tumor apoptotic and metastatic factors on mRNA levels by quantitative PCR and protein levels by western blotting revealed that these phytochemical treatments effectively negated nicotine-induced upregulated expression of bcl-2, bcl-2/bax ratio, caspase-3, matrix metalloproteinase (MMP)-2 and MMP-9 as well as downregulated expression of bax. Further analysis of inflammatory markers such as tumor necrosis factor (TNF)- $\alpha$ and interleukin (IL)-6 in cell culture supernatant and mRNA and protein expression of nuclear transcription factor-kappaB $(\mathrm{NF}-\kappa \mathrm{B})$ and I kappa B-alpha (IкB- $\alpha$ ) was carried out to substantiate the anti-inflammatory effect of flavones in Scutellaria in nicotine-exposed lung cancer cells. The therapeutic effects observed in the present study are attributed to the potent potential against proliferation, metastasis and inflammatory
\end{abstract}

Correspondence to: Professor Jingcheng Dong, Institute of Integrative Medicine, Department of Integrative Medicine, Huashan Hospital, Fudan University, 12 Middle Wulumuqi Road, Shanghai 200041, P.R. China

E-mail: jcdong2004@126.com

Key words: Scutellaria baicalensis, lung cancer, nicotine, metastasis, inflammation microenvironment by flavonoid components in Scutellaria in nicotine-induced lung cancer cells.

\section{Introduction}

Lung cancer is the most common cancer in the world (1). It is the leading cause of cancer-related mortalities in China, with a rate of about 470,000 people in 2005 (2). The causal relation between smoking and lung cancer has been unambiguously established (3). Over $80 \%$ of lung cancer cases are caused by cigarette smoking. In spite of tobacco control activities and policies, the prevalence of smoking in China is still high, with 350 million smokers and 740 million passive smokers (4). Therefore, treatment of cigarette smoke-induced diseases is essential.

Among the 4,000 identified chemicals in cigarette smoke, nicotine is the reason why people continue to smoke despite the obvious side effects on health. Nicotine acts through nicotinic acetylcholine receptors ( $\mathrm{AAChRs)}$ that are widely present on neuronal and non-neuronal cells $(5,6)$. At the end of last century, Maneckjee and Minna, firstly reported that lung cancer cell lines also expressed nAChRs $(7,8)$. Subsequently, studies have shown that nAChRs subunits are expressed on biopsy specimens of lung cancer (9). The main effects of nicotine on lung cancer cells involve promotion of cell proliferation and prevention of drug-induced apoptosis. B-cell lymphoma-2 (Bcl-2) protein family plays a vital role in regulating apoptosis and activating the subsequent caspase cascade $(10,11)$. The process of apoptosis is regulated by a complicated interaction between proapoptotic (Bcl-2-associated $\mathrm{X}$ protein, $\mathrm{Bax}$ ) and anti-apoptotic (Bcl-2) proteins. Bax is an essential component in the nicotine survival signaling pathway, through activating PI3K/AKT that phosphorylates and inactivates the pro-apoptotic function of Bax (12). In addition, nicotine facilitates invasion and metastasis of lung cancer $(13,14)$. Matrix metalloproteinases (MMPs) have long been linked with cancer cell invasion and metastasis (15). Matrix metalloproteinase-2 (MMP-2) and matrix metalloproteinase-9 (MMP-9) are main members of MMPs and patients with high MMP-2 or MMP-9 expression have poorer overall survival (16-18). Nicotine increased the activity of MMP-2 in esophageal squamous 
carcinoma cells and epithelial-mesenchymal transition (EMT) in lung cancer cells $(13,19)$. Thus, though nicotine itself cannot provoke cancer, it contributes to lung cancer progression already initiated through diverse processes.

Scutellaria baicalensis, one of the most popular and multi-purpose herbs used in China, possess potent anticancer activities. The major constituents of Scutellaria are baicalin, baicalein and wogonin, the bioactive flavones. These phytochemicals are cytotoxic to various lung cancer cell lines and suppress tumor growth in vivo without systemic toxicity $(20,21)$. The antitumor functions of these flavones are primarily due to their abilities to induce cell cycle arrest, regulate apoptosis-related proteins expression, eliminate adhesion and migration capacities and inhibit metastasis $(22,23)$. Previous studies from our laboratory have shown that baicalin attenuated cigarette smoke-induced pulmonary chronic inflammation, declined inflammatory cells as well as tumor necrosis factor (TNF)- $\alpha$, interleukin (IL)-6, IL-8 and MMP-9 production, decreased nuclear transcription factor-kappaB (NF- $\mathrm{kB}) \mathrm{p} 65$ expression in the lung, and inhibited the activation of NF- $\mathrm{KB}(24,25)$. Pulmonary chronic inflammation and lung cancer are closely correlated. Many common features coexist in both diseases, and the potential shared biological mechanisms are inflammation, EMT and others, especially the inflammatory factor $(26,27)$. Recent reports demonstrated that tobacco smoke promoted lung tumorigenesis by triggering inflammatory pathways (28).

In view of these observations, we hypothesized that flavones in Scutellaria may inhibit nicotine-induced lung cancer progression. The present study was designed to evaluate the antitumor effects and underlying mechanisms of baicalin, baicalein and wogonin on nicotine-induced lung cancer development in vitro. The results of this study may help us designing novel therapies based on these biosafe agents that target nicotine-induced proliferation, metastasis and inflammation in lung cancer cells.

\section{Materials and methods}

Materials. Baicalin, baicalein and wogonin were purchased from Shanghai Ronghe Corp. (Shanghai, China). Nicotine (liquid, N3876) was purchased from Sigma-Aldrich (St. Louis, MO, USA). CCK-8 kit was purchased from Dojindo Moleculare Technologies, Inc. (Kumamoto, Japan). Bcl-2, bax, caspase-3, NF- $\kappa B$ p 65 and I $\kappa B-\alpha$ monoclonal antibodies were purchased from Beyotime Institute of Biotechnology (Haimen, China). MMP-2 and MMP-9 monoclonal antibodies were purchased from ABGENT Corp. (San Diego, CA, USA). IL-6 and TNF- $\alpha$ Human ELISA kits were purchased from Life Technologies Corp. (Grand Island, NY, USA).

Cell culture and treatments. A549 and H1299 cells were obtained from Chinese Academy of Sciences (Shanghai, China) and maintained in DMEM high glucose culture medium supplemented with $10 \%$ fetal bovine serum, $100 \mathrm{U} / \mathrm{ml}$ penicillin and $100 \mu \mathrm{g} / \mathrm{ml}$ streptomycin at $37^{\circ} \mathrm{C}$ in humidified atmosphere with $5 \% \mathrm{CO}_{2}$. All cell culture reagents were purchased from Hyclone Laboratories (Logan, UT, USA).

A549 and H1299 cells were grown to 70-80\% confluency in $100-\mathrm{mm}$ culture plates in a total volume of $5 \mathrm{ml}$ DMEM medium containing $10 \%$ fetal bovine serum (FBS). Cells were then serum-starved for $12 \mathrm{~h}$ and washed with ice-cold sterile PBS before being treated with nicotine $\left(10^{-6} \mathrm{M}\right.$ for A549 cells and $10^{-8} \mathrm{M}$ for H1299 cells) combined with or without flavones. Baicalin, baicalein or wogonin, dissolved in dimethyl sulfoxide (DMSO), were used for the treatment of cells. The final concentration of DMSO used was $<0.1 \%(\mathrm{v} / \mathrm{v})$. For treatment with baicalin $(50 \mu \mathrm{M})$, baicalein $(10 \mu \mathrm{M})$ or wogonin $(1 \mu \mathrm{M})$, cells were incubated for $48 \mathrm{~h}$.

Viability assays. Cell viability in A549 and H1299 cells was measured by the CCK-8 assay kit following the manufacturer's instructions. Briefly, A549 and H1299 cells were seeded at a density of 5,000 cells/100 $\mu \mathrm{l} /$ well in 96 -well culture plates in serum-free media and incubated in a humidified incubator at $37^{\circ} \mathrm{C} 12 \mathrm{~h}$ prior to treatment with nicotine with or without baicalin, baicalein or wogonin as indicated above. After incubation, $10 \mu \mathrm{l} \mathrm{CCK-8}$ was added to each well for $2 \mathrm{~h}$ after which the optical density (OD) was measured at $490 \mathrm{~nm}$. The percent of viable cells was determined by the formula: ratio $(\%)=$ [OD (baicalin/baicalein/wogonin + nicotine) - OD (Blank)] / [OD(Control) - OD (Blank)] x $100 \%$. The cell viability data are averages of 3 independent experiments each containing 3 replicates.

Flow cytometric analysis. After the treatment of A549 and H1299 cells with nicotine with or without baicalin, baicalein or wogonin as mentioned above, $1 \times 10^{6}$ cells were harvested and washed once with binding buffer (HEPES buffer: $10 \mathrm{mM}$ HEPES/NaOH, pH 7.4, $150 \mathrm{mM} \mathrm{NaCl}, 5 \mathrm{mM} \mathrm{KCl}, 1 \mathrm{mM}$ $\mathrm{MgCl}_{2}, 1.8 \mathrm{mM} \mathrm{CaCl} 2$ ). After aspiration of the supernatant, cells were resuspended in $100 \mu \mathrm{l}$ binding buffer containing $1 \mu \mathrm{l}$ Annexin V-FITC conjugated antibody and $5 \mu \mathrm{l}$ PI for exactly $5 \mathrm{~min}$ in the dark at room temperature. Cells were than analyzed on a FACSCalibur cytometer (Becton-Dickinson, San Jose, CA, USA). The data were analyzed using FlowJo software V6.0 (Tree Star, Ashland, OR, USA).

Wound healing assay. Cells were cultured on 6-well plates $\left(3 \times 10^{5}\right.$ cells/well) and treated as indicated above. When confluent, the monolayers were scratched horizontally with a yellow pipette tip to obtain a monolayer culture with space without cells. Media and dislodged cells were aspirated. The cells were incubated along with nicotine with or without baicalin, baicalein or wogonin as above. After incubation, cell invasion was observed; three randomly fields along the scraped line were selected, and images were photographed on each well using a phase contrast inverted microscope.

Invasion assay. In vitro cell invasion was performed by the $6.5 \mathrm{~mm}$ Transwell ${ }^{\circledR}$ with an $8.0-\mu \mathrm{m}$ pore polycarbonate membrane insert (Corning Co., USA). Matrigel was purchased from $\mathrm{BD}$ Biosciences and stored at $-20^{\circ} \mathrm{C}$. After thawing at $4^{\circ} \mathrm{C}$ overnight, the matrigel was diluted in serum-free DMEM medium; $50 \mu 1$ of the diluted matrigel were evenly inoculated into the upper chamber of the $6.5 \mathrm{~mm}$ Transwell membrane and allowed to form a gel at $37^{\circ} \mathrm{C}$. Cells $\left(1 \times 10^{6}\right)$ suspended in $250 \mu \mathrm{l}$ of serum-free DMEM were seeded into the upper compartments of each chamber in the presence of nicotine with or without baicalin, baicalein or wogonin as previously 
Table I. Primers used for real-time quantitative PCR analysis.

\begin{tabular}{ll}
\hline Sequence name & \multicolumn{1}{c}{ Primers } \\
\hline$\beta$-actin-F & 5'-CCTGTACGCCAACACAGTGC-3' \\
$\beta$-actin-R & 5'-ATACTCCTGCTTGCTGATCC-3' \\
Bcl-2-F & 5'-CCAGGCCGGCGACGACTTCTC-3' \\
Bcl-2-R & 5'-ATCTCCCGGTTGACGCTCTCCACA-3' \\
Bax-F & 5'-GGTTGTCGCCCTTTTCTACTT-3' \\
Bax-R & 5'-TGAGCACTCCCGCCACAA-3' \\
Pro-caspase-3-F & 5'-GTGGAATTGATGCGTGATGTT-3' \\
Pro-caspase-3-R & 5'-GGCAGGCCTGAATAATGAAA-3' \\
MMP-2-F & 5'-CACGCTGGGCCCTGTCACTCCT-3' \\
MMP-2-R & 5'-TGGGGCCTCGTATACCGCATCAAT-3' \\
MMP-9-F & 5'-TGCCCGGACCAAGGATACAGTTT-3' \\
MMP-9-R & 5'-AGGCCGTGGCTCAGGTTCAGG-3' \\
P-p65-F & 5'-CTCCGCGGGCAGCATCC-3' \\
P-p65-R & 5'-CATCCCGGCAGTCCTTTCCTACAA-3' \\
IкB- $\alpha-F$ & 5'-CACCCCGCACCTCCACTCCATC-3' \\
IкB- $\alpha-R$ & 5'-ACATCAGCCCCACACTTCAACAGG'
\end{tabular}

indicated, whereas the lower compartments were filled with $500 \mu \mathrm{l}$ of DMEM with $10 \%$ FBS. After incubation, the noninvasive cells were removed from the upper surface of the membrane by scrubbing. Cells on the reverse side were stained with $0.1 \%$ crystal violet, and invasive cells were counted under a microscope at $\mathrm{x} 400$ magnification.

Cytokine analysis. TNF- $\alpha$ and IL-6 levels in A549 and H1299 cell culture supernatant were measured using ELISA kits in accordance with the manufacturer's recommendations.

Real-time quantitative polymerase chain reaction (PCR). Total RNA was isolated from A549 and H1299 cells using TRIzol reagent (Gibco BRL, Gaithersburg, MD, USA). The mRNA levels were analyzed by real-time quantitative RT-PCR using SYBR Premix Ex Taq System (Takara, Dalian, China). mRNA was reverse-transcribed into cDNA by cDNA synthesis kit (Takara). Specific primers (Table I) were designed to screen the expression of bcl-2, bax, pro-caspase-3, MMP-2,

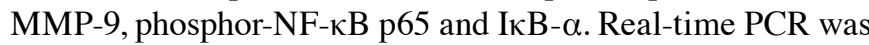
performed using a SYBR Premix Ex Taq kit (Takara) and run for the denaturation step at $94^{\circ} \mathrm{C}$ for $10 \mathrm{sec}$, the annealing at $58-60^{\circ} \mathrm{C}$ for $20 \mathrm{sec}$ and the extension at $72^{\circ} \mathrm{C}$ for $20 \mathrm{sec}$. The final extension was at $72^{\circ} \mathrm{C}$ for $5 \mathrm{~min}$. The annealing temperature was $58^{\circ} \mathrm{C}$ for bax and pro-caspase- 3 and $60^{\circ} \mathrm{C}$ for bcl-2, MMP-2, MMP-9, NF- $\kappa$ B p65 and I $\kappa$ - $\alpha$. Each cDNA sample was run in triplicate and the corresponding non-real-time mRNA sample was included as a negative control. The primers of $\beta$-actin were included in every plate to avoid sample variations. The mRNA level of each sample for each gene was normalized to that of $\beta$-actin mRNA and gene expression changes induced by various treatments were determined by the $2^{-\Delta \Delta C T}$ method (29). For the validation, real-time PCR was performed 3 times independently.

Western blotting. The extraction of cytosolic and nuclear proteins of the cells was performed according to instructions of protein extraction kit (Beyotime Biotechnology, Haimen, China). Protein concentrations were determined by BCA protein assay kit (Beyotime). Equal amounts of protein were separated by $8 \%$ SDS-polyacrylamide gel electrophoresis, and transferred to polyvinylidene difluoride (PVDF) membranes. The membranes were then blocked at room temperature for $1.5 \mathrm{~h}$ with $5 \%(\mathrm{w} / \mathrm{v})$ non-fat milk in TBST buffer and incubated with primary antibodies in TBST overnight at $4^{\circ} \mathrm{C}$ with continuous shaking. After three washes in TBST, membranes were incubated with secondary antibodies conjugated with horseradish peroxidase for $1 \mathrm{~h}$ and visualized by enhanced chemiluminescence using Supersignal West Femto Chemiluminescent Substrate (Pierce Biotechnology Inc., Rockford, IL, USA). Band intensities were quantified using UN-SCAN-IT gel analysis software (version 6). The optical density for target protein was shown as a proportion of $\beta$-actin optical density. The western blot data were replicated 3 times.

Statistical analysis. Data are expressed as mean \pm SEM. Statistically significant differences between groups were determined by ANOVA followed by Bonferroni's post hoc comparison tests. All analyses were undertaken using the statistical software SPSS 18.0. A value of $\mathrm{P}<0.05$ was considered statistically significant.

\section{Results}

Flavones in Scutellaria inhibit nicotine-induced cell viability. The cell viability of flavones in Scutellaria treatment on nicotine-induced lung cancer cells (A549 and H1299) were determined by CCK-8 assay (Fig. 1). Exposure to nicotine increased cell proliferation in both lung cancer cell lines; baicalin, baicalein and wogonin all significantly inhibited nicotine-induced A549 cell proliferation, while only baicalein and wogonin decreased nicotine-induced H1299 cell proliferation.

Flavones in Scutellaria inhibit nicotine-induced cell apoptosis. To determine whether the cytotoxicity of flavones in Scutellaria on nicotine-induced lung cancer cells occurred by apoptosis, we measured the percentage of Annexin V-positive and PI-negative cells in each group (Fig. 2). Treatment with baicalin, baicalein or wogonin along with nicotine for $48 \mathrm{~h}$ resulted in an increased number of early-stage apoptotic A549 cells to $6.90,19.63$ and $22.50 \%$ and $\mathrm{H} 1299$ cells to $10.67,10.22$ and $10.07 \%$ respectively, compared with $3.30 \%$ in A549 cells and $2.50 \%$ in H1299 cells only treated with nicotine.

Flavones in Scutellaria inhibited nicotine-induced cell invasion. To examine the effect of flavones in Scutellaria on nicotine-induced invasion of lung cancer cells, Transwell membrane coated with Matrigel was utilized. The results showed that the number of both A549 and H1299 cells invaded to the lower chamber was significantly increased by a $48 \mathrm{~h}$ treatment of nicotine, while the number of invading cells was apparently reduced through the Matrigel membrane in groups of baicalin, baicalein or wogonin along with nicotine (Fig. 3).

Flavones in Scutellaria inhibit nicotine-induced woundhealing migratory ability. We performed the wound-healing 
A

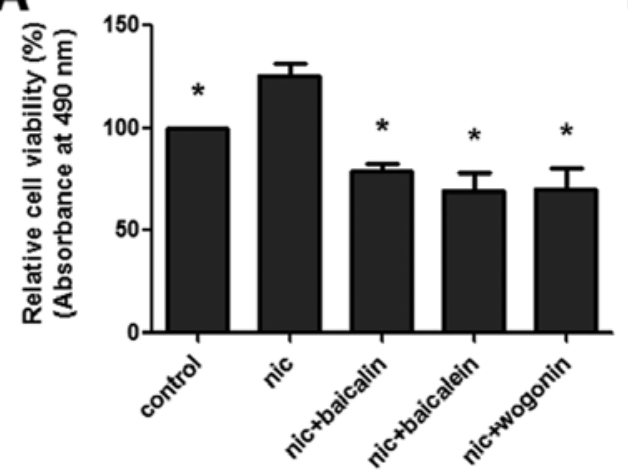

B

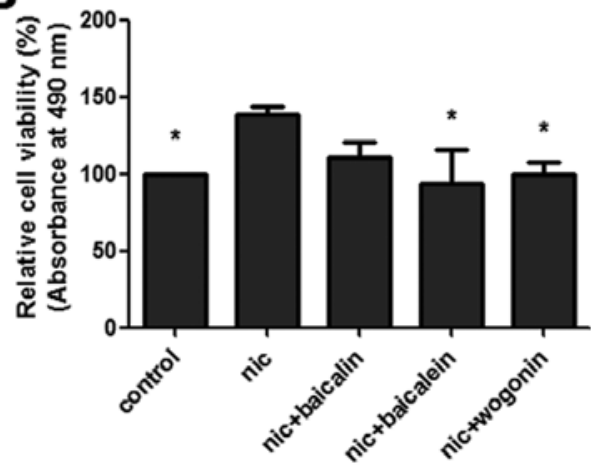

Figure 1. Nicotine induces proliferation in A549 and H1299 cells, which is downregulated by flavones in Scutellaria. A549 (A) and H1299 cells (B) were treated with nicotine and/or baicalin, baicalein or wogonin for $48 \mathrm{~h}$ for CCK- 8 assay. Data are the average of 3 independent experiments each containing 3 replicates. ${ }^{*} \mathrm{P}<0.05$ vs. nicotine group.

A

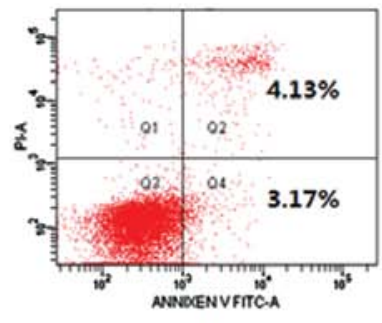

control

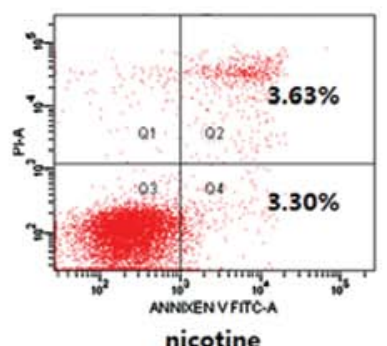

nicotine

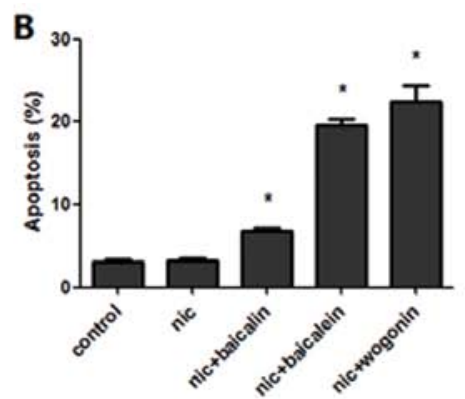



nicotine+baicalin

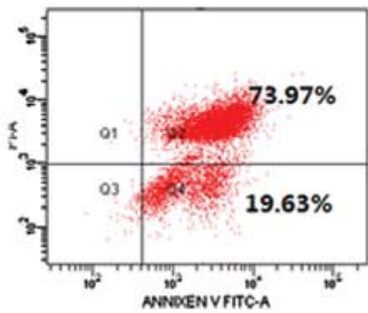

nicotine+baicalein

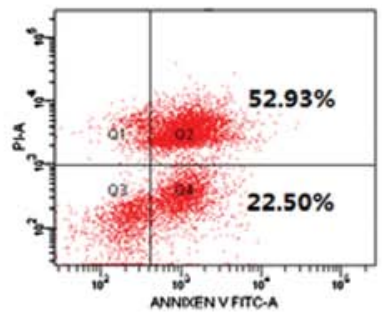

nicotine+wogonin
C

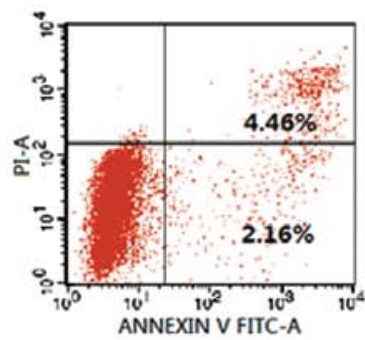

control

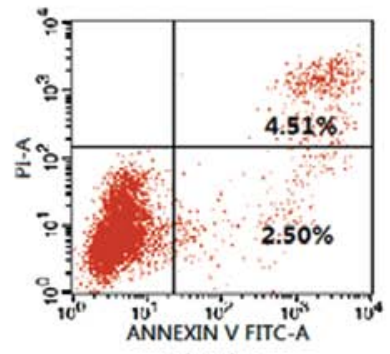

nicotine
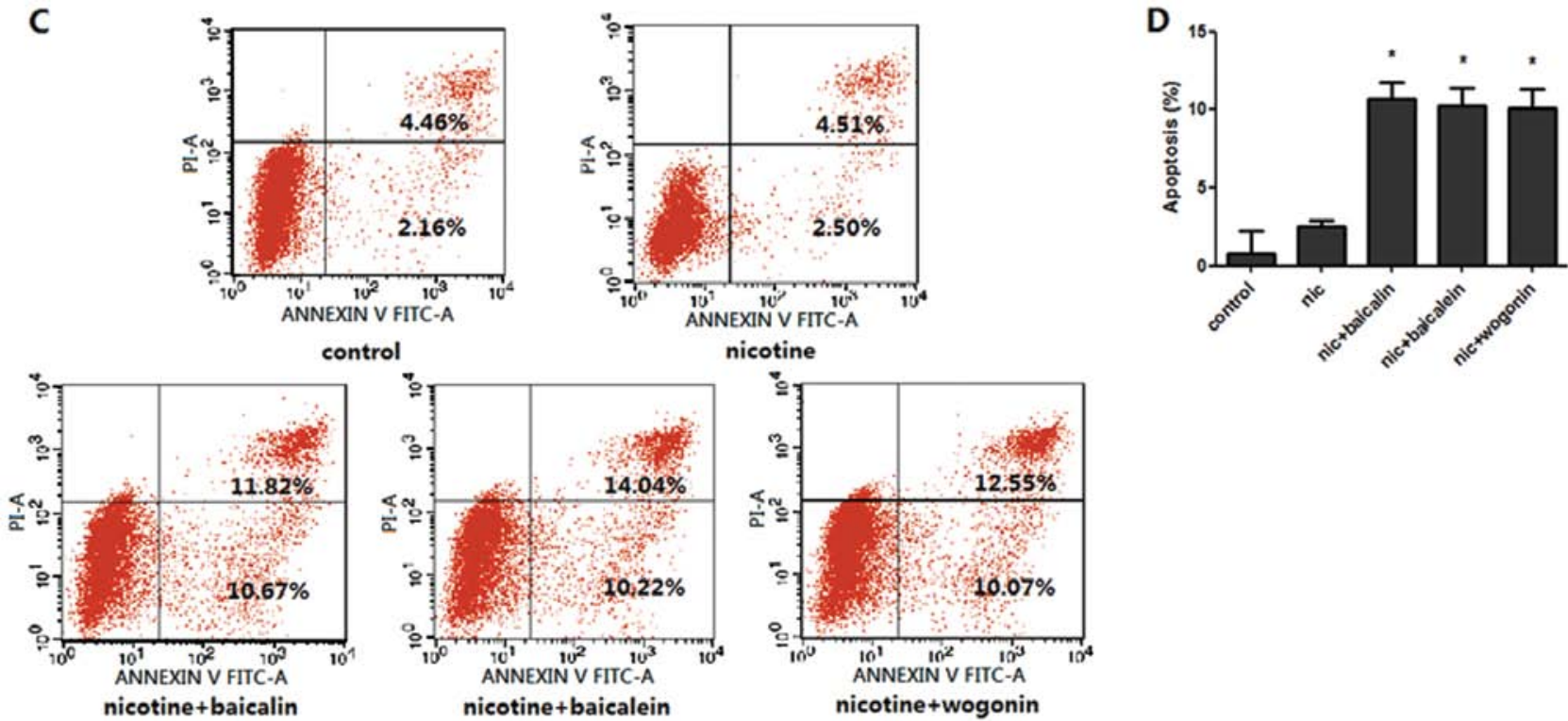

Figure 2. Flavones in Scutellaria induce apoptosis of nicotine-exposed A549 and H1299 cells. Scatter plot of apoptotic A549 (A) and H1299 cells (C) treated with nicotine and/or baicalin, baicalein or wogonin for $48 \mathrm{~h}$ for flow cytometry analysis. Early apoptotic cells are defined as Annexin $\mathrm{V}^{+} / \mathrm{PI}^{-}$, whereas late apoptotic/necrotic cells are Annexin $\mathrm{V}^{+} / \mathrm{PI}^{+}$. Data are representative results for 1 of the 3 replicates. Percentage of Annexin $\mathrm{V}^{+} / \mathrm{PI}{ }^{-} \mathrm{A} 549$ (B) and $\mathrm{H} 1299$ cells $(\mathrm{D})$ treated with nicotine and/or baicalin, baicalein or wogonin for $48 \mathrm{~h}$. Data are the average of 3 independent experiments each containing 3 replicates. ${ }^{*} \mathrm{P}<0.05$ vs. nicotine group. 
A

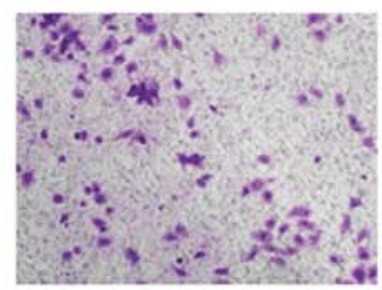

control

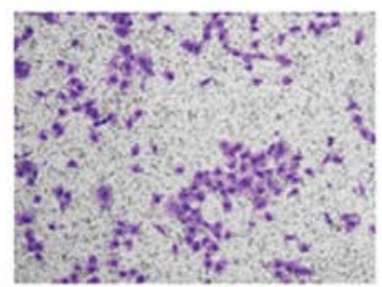

nicotine
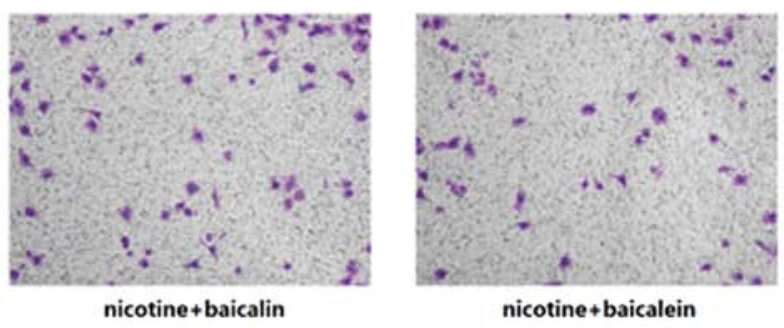

nicotine+baicalein

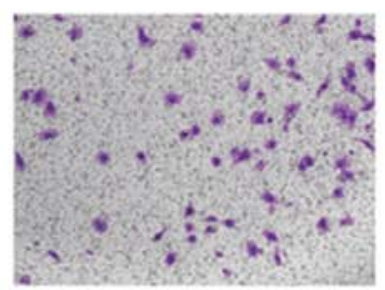

nicotine + wogonin
C
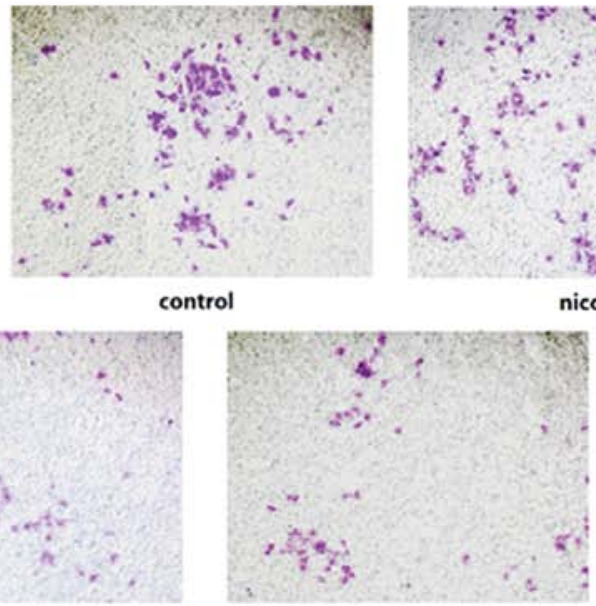

nicotine+baicalein

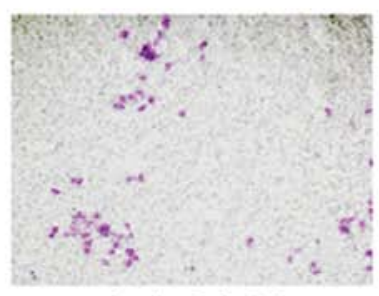

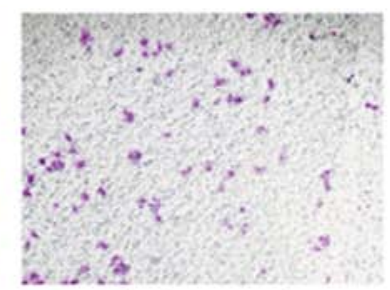

nicotine+wogonin
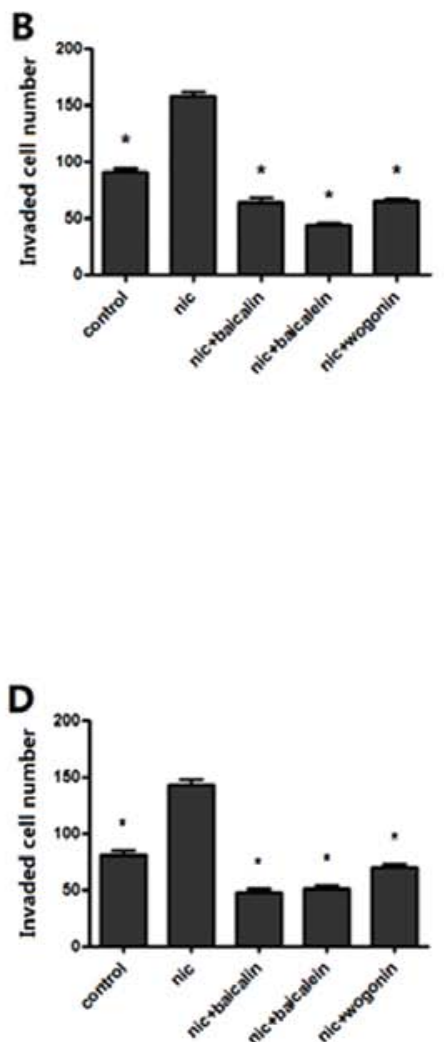

Figure 3. Flavones in Scutellaria block nicotine-enhanced lung cancer cell invasion. A549 (A) and H1299 cells (C) treated with nicotine and/or baicalin, baicalein or wogonin for $48 \mathrm{~h}$ for invasion assay. Number of invaded A549 (B) and H1299 cells (D) treated with nicotine and/or baicalin, baicalein or wogonin. Data are the average of 3 independent experiments each containing 3 replicates. ${ }^{*} \mathrm{P}<0.05$ vs. nicotine group.

assay to determine the effect of flavones in Scutellaria on nicotine-induced migration of lung cancer cell. Compared with the control, an obvious increase of cells in the denuded zone was observed at the A549 cells treated with nicotine for 48 h (Fig. 4A). A549 cells exposed to baicalin, baicalein or wogonin along with nicotine expressed a decreased ability to migrate as compared to the nicotine group. The quantitative data revealed that these phytochemicals could inhibit the nicotine-induced migration of A549 cells (Fig. 4B). The effect of nicotine on $\mathrm{H} 1299$ cells for $72 \mathrm{~h}$ was similar to that on A549 cells, while data demonstrated that only baicalein negated nicotine-induced migration of H1299 cells (Fig. 4C and D).

Flavones in Scutellaria inhibit nicotine-induced TNF- $\alpha, I L-6$ levels. To test the effect of flavones in Scutellaria on nicotineinduced lung cancer-associated inflammation, TNF- $\alpha$ and IL-6 expressions were measured by ELISA for the supernatant of A549 and H1299 cells treated with nicotine combined with or without various flavones. Treatment with nicotine caused a significant augment in the levels of TNF- $\alpha$ and IL- 6 expression in both A549 and H1299 cells; nicotine with baicalin, baicalein or wogonin treated A549 and H1299 cells reduced
TNF- $\alpha$ expression and the same treated A549 cell decreased IL-6 expression, whereas only nicotine plus baicalin or baicalein showed a decreased expression of IL-6 in H1299 cells (Fig. 5).

Flavones in Scutellaria modulate nicotine-induced bcl-2 family and caspase-3. Anti-apoptotic effect of flavones in Scutellaria on nicotine-induced cells prompted us to examine whether bcl-2 family and caspase-3 plays any role in the model. The mRNA level of bcl-2 was increased in both A549 and H1299 cells treated with nicotine, whereas treatment with nicotine along with baicalin, baicalein or wogonin sharply decreased the bcl-2 mRNA level. Nicotine treatment increased bax mRNA level in H1299 cells but not in A549 cells; treatment with nicotine and baicalin decreased bax mRNA level in both cell lines, treatment with nicotine and baicalein only decreased the level of bax mRNA in H1299 cells, while nicotine and wogonin caused an increased bax mRNA level in A549 cells but a reduced level in H1299 cells (Figs. 6A and 7A). The above changes had the effect of greatly increasing the messenger ratio of the anti-apoptotic bcl-2 to the proapoptotic bax in A549 and H1299 cells treated with 

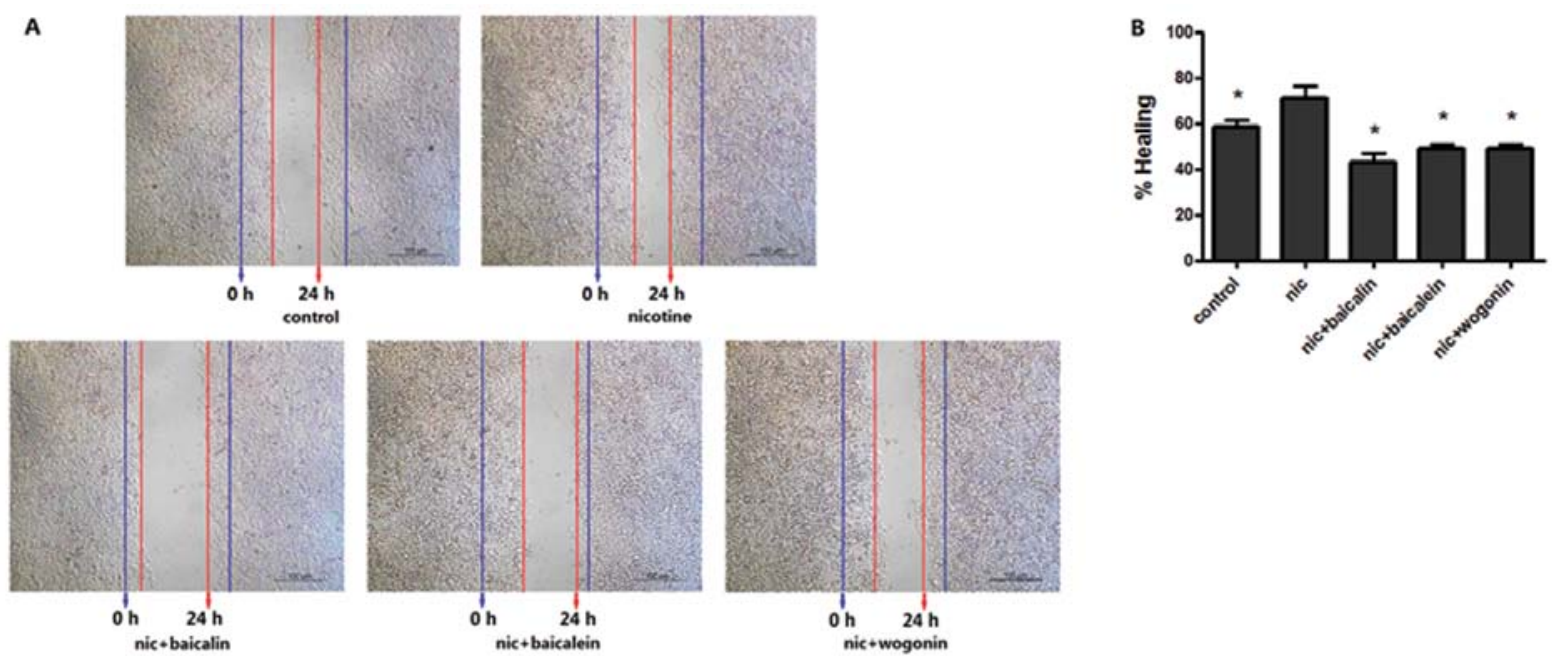

C
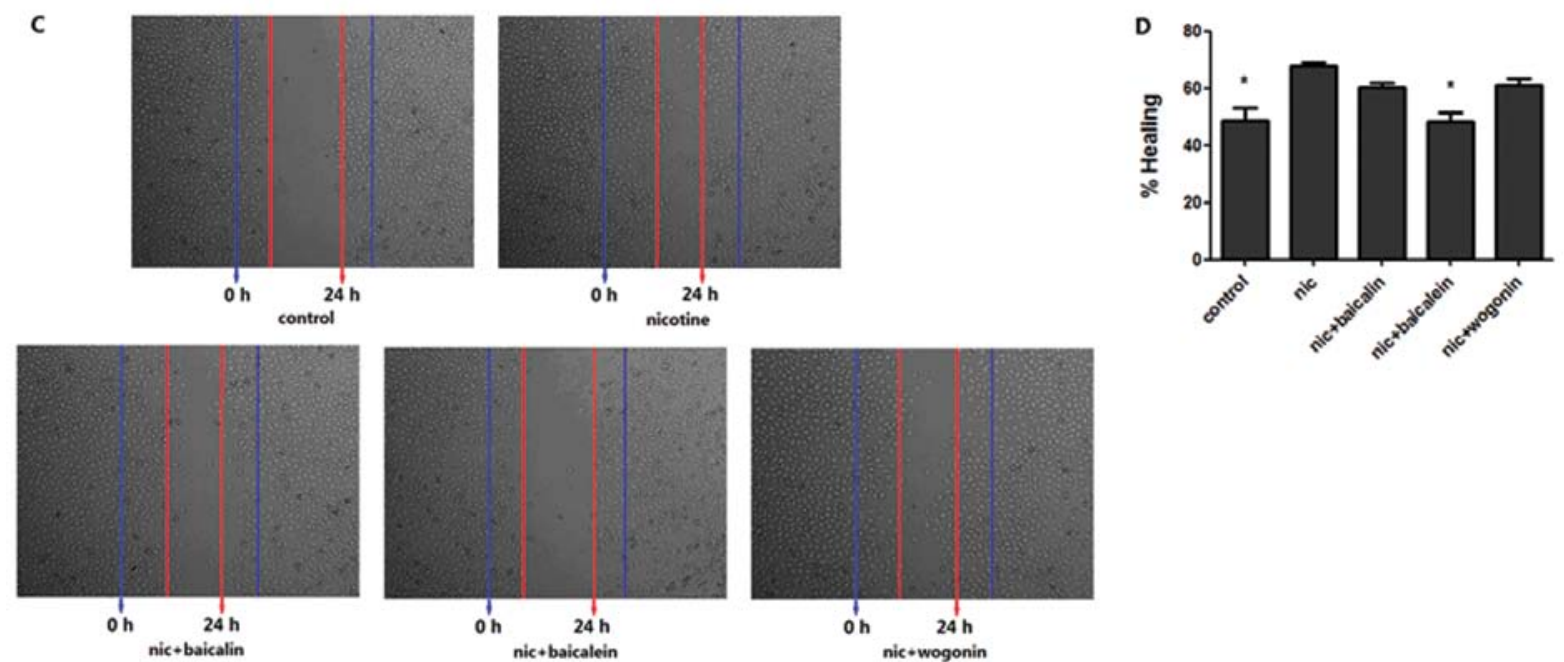

Figure 4. Flavones in Scutellaria inhibit nicotine-enhanced lung cancer cell migration. A confluent A549 (A) and H1299 cell (C) monolayer was wounded with a yellow pipette tip and then treated with nicotine and/or baicalin, baicalein or wogonin for 48 or $72 \mathrm{~h}$, respectively, for wound-healing assay. Percentage of healing A549 (B) and H1299 cells (D) treated with nicotine and/or baicalin, baicalein or wogonin. Data are the average of 3 independent experiments each containing 3 replicates. ${ }^{*} \mathrm{P}<0.05$ vs. nicotine group.


Figure 5. Flavones in Scutellaria attenuate nicotine-induced TNF- $\alpha$ and IL-6 levels in lung cancer cells. TNF- $\alpha$ and IL-6 levels in A549 (A and B) and H1299 cells $(\mathrm{C}$ and $\mathrm{D})$ were measured by ELISA. Data are the average of 3 independent experiments each containing 3 replicates. ${ }^{*} \mathrm{P}<0.05$ vs. nicotine group. 

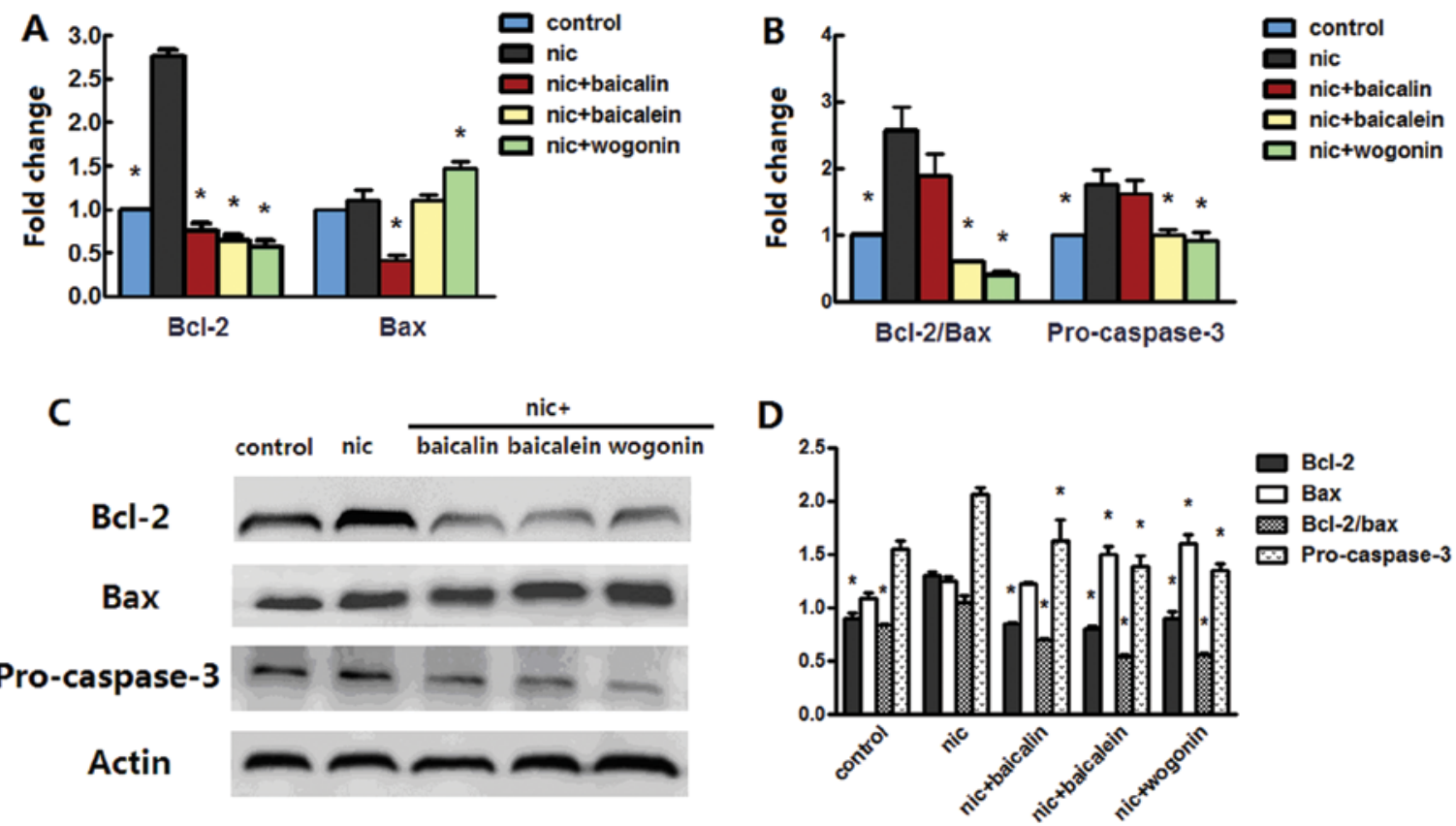

Figure 6. Flavones in Scutellaria modulated nicotine-induced mRNA and protein expressions of bcl-2, bax, bcl-2/bax and pro-caspase-3 in A549 cells. The mRNA expressions of bcl-2, bax (A), bcl-2/bax and pro-caspase-3 (B) were measured by quantitative real-time PCR. The protein expressions of bcl-2, bax, bcl-2/bax and pro-caspase-3 were measured by western blotting (C). Band intensities were quantified using UN-SCAN-IT gel analysis software (version 6). The optical density for target protein was shown as a proportion of $\beta$-actin optical density (D). " $\mathrm{P}<0.05$ vs. nicotine group.

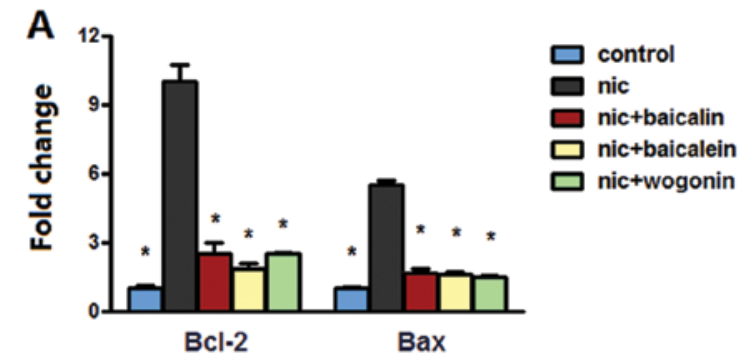

C

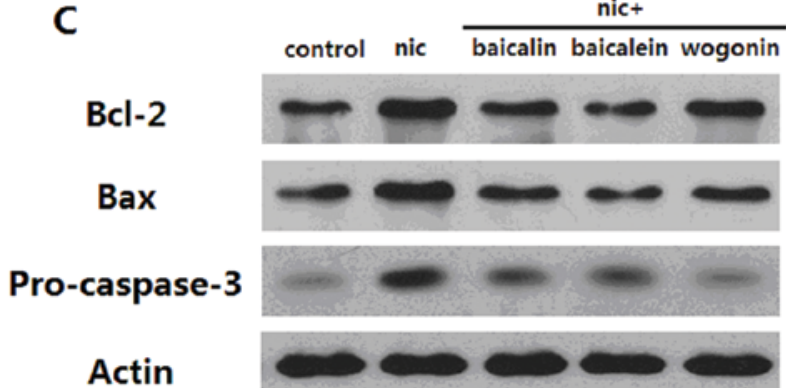

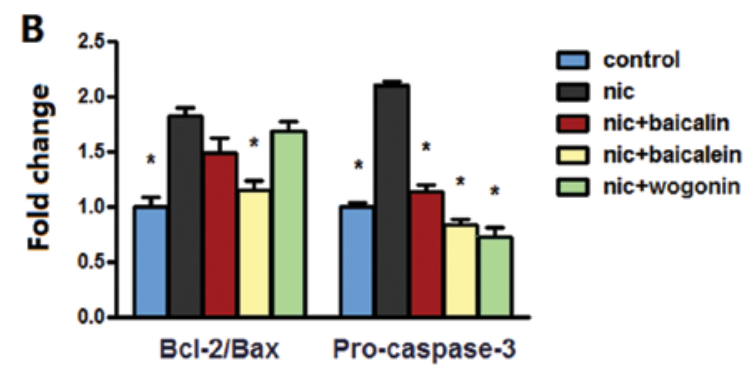

D

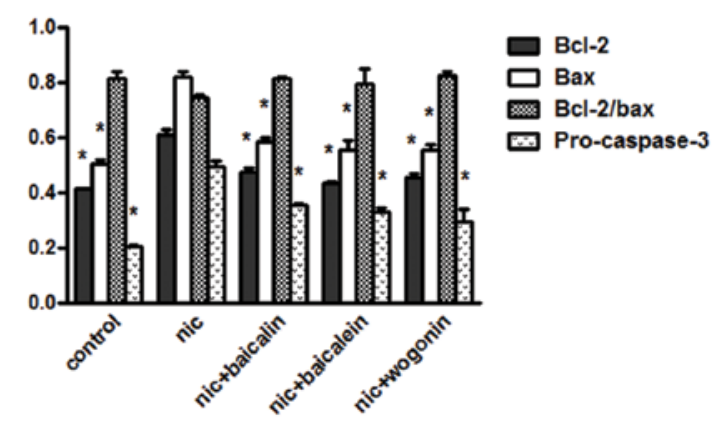

Figure 7. Flavones in Scutellaria modulate nicotine-induced mRNA and protein expressions of bcl-2, bax, bcl-2/bax and pro-caspase-3 in H1299 cells. The mRNA expressions of bcl-2, bax (A), bcl-2/bax and pro-caspase-3 (B) were measured by quantitative real-time PCR. The protein expression of bcl-2, bax, bcl-2/bax and pro-caspase-3 was measured by western blotting (C). Band intensities were quantified using UN-SCAN-IT gel analysis software (version 6). The optical density for the target protein is shown as a proportion of $\beta$-actin optical density (D). ${ }^{*} \mathrm{P}<0.05$ vs. nicotine group.

nicotine; the ratio decreased in nicotine along with baicalein or wogonin treated A549 cells and in nicotine and baicalein treated H1299 cells. Nicotine treatment greatly increased pro-caspase-3 mRNA level in both cell types, while addition of baicalein or wogonin in A549 cells or addition of baicalin, baicalein or wogonin in H1299 cells significantly abrogated this effect (Figs. 6B and 7B). Western blot analysis of bcl-2 and bax of both cells treated with nicotine reconfirmed the results in real-time PCR; in groups receiving nicotine with baicalin, baicalein or wogonin, protein expressions of bcl-2 in A549 and H1299 cells and the expression of bax in H1299 cells was dramatically reduced, and in groups receiving nicotine with 

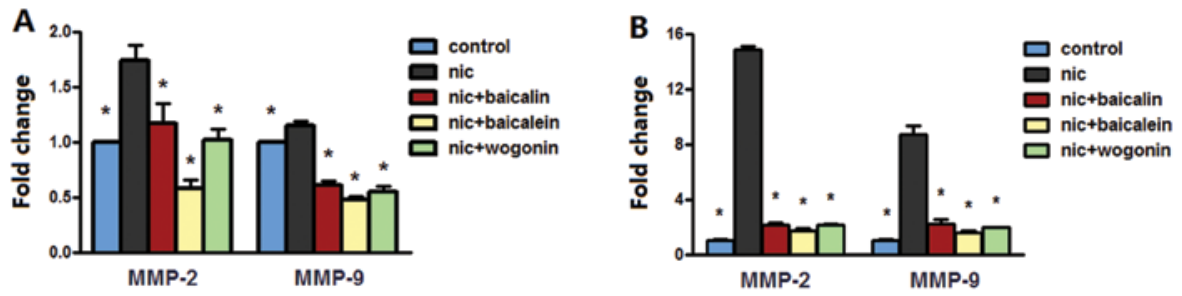

C

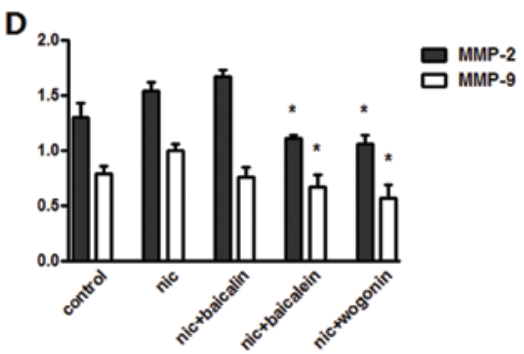

$\mathbf{E}$

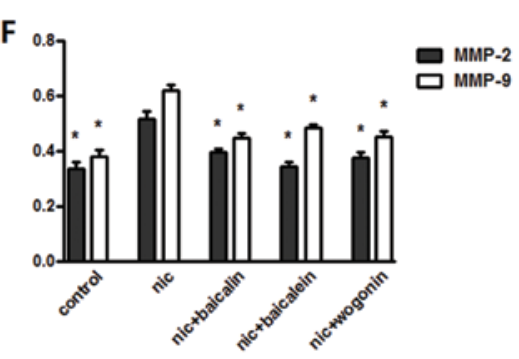

Figure 8. Flavones in Scutellaria inhibit nicotine-induced mRNA and protein expression of MMP-2 and MMP-9 in A549 and H1299 cells. The mRNA expression of MMP-2 and MMP-9 was measured by quantitative real-time PCR in A549 (A) and H1299 cells (B). The protein expressions of MMP-2 and MMP-9 in A549 (C) and H1299 cells (E) was measured by western blotting. Band intensities were quantified using UN-SCAN-IT gel analysis software (version 6). The optical density for the target protein is shown as a proportion of $\beta$-actin optical density [(D) for A549 cells and (F) for H1299 cells]. ${ }^{*} \mathrm{P}<0.05$ vs. nicotine group.

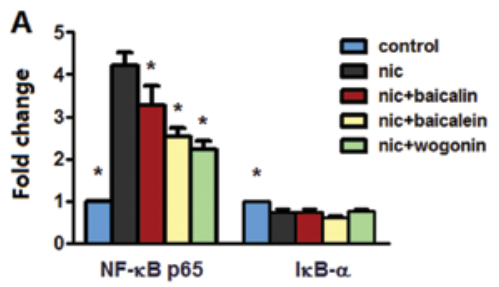

C

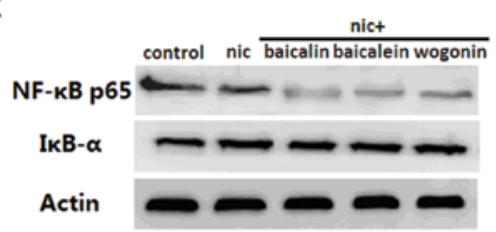

$\mathbf{E}$

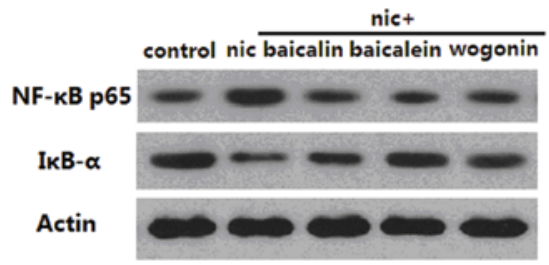

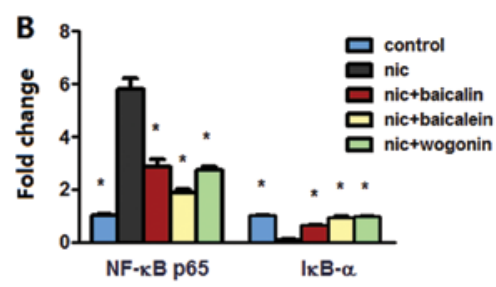
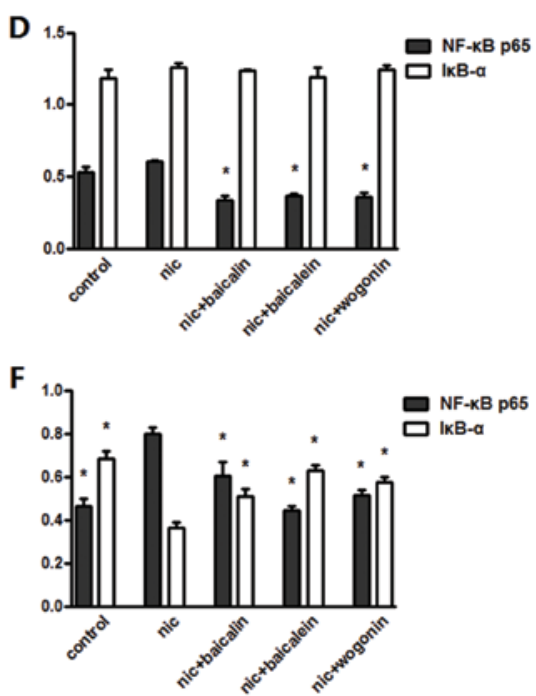

Figure 9. Flavones in Scutellaria modulate nicotine-induced mRNA and protein expressions of NF- $\kappa$ B $p 65$ and IкB- $\alpha$ in A549 and H1299 cells. The mRNA

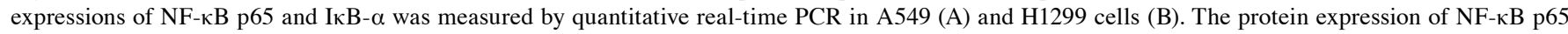
and IкB- $\alpha$ in A549 (C) and H1299 cells (E) was measured by western blotting. Band intensities were quantified using UN-SCAN-IT gel analysis software (version 6). The optical density for the target protein is shown as a proportion of $\beta$-actin optical density [(D) for A549 cells and (F) for H1299 cells]. ${ }^{*} \mathrm{P}<0.05$ vs. nicotine group. 
baicalein or wogonin, the expression of bax in A549 cells was increased. These changes caused upregulation of bcl-2/ bax protein expression in nicotine group and downregulation of the ratio in nicotine along with baicalin, baicalein or wogonin treated A549 cells but no appreciable ratio changes were observed in H1299 cells. Nicotine treatment induced procaspase-3 aggregation in H1299 cells but not in A549 cells, while each of flavones with nicotine treatment diminished this aggregation in both cell lines (Figs. 6C and D and 7C and D).

Flavones in Scutellaria inhibit nicotine-induced MMP-2 and MMP-9. To understand the mechanism underlying the suppression of nicotine-induced migration and invasion by phytochemicals in Scutellaria, we checked the modulation in MMP-2 and MMP-9 mRNA and protein levels. Results of real-time PCR assay depicted upregulation of MMP-2 and MMP-9 mRNA upon nicotine treatment, whereas these effects were reversed by treatments of nicotine with baicalin, baicalein or wogonin in A549 and H1299 cells (Fig. 8A and B). Western blot analysis showed that nicotine-exposed H1299 cells but not A549 cells augmented the MMP-2 and MMP-9 protein expression; nicotine with baicalein or wogonin inhibited nicotine-induced MMP-2 and MMP-9 expression in both cell lines, and nicotine and baicalin treatment only prevented nicotine-induced MMP-2 and MMP-9 expression in H1299 cells (Fig. 8C-F).

Flavones in Scutellaria modulate nicotine-induced $N F-\kappa B$ $p 65$ and $I \kappa B-\alpha$. We searched for the mechanisms that may be responsible for inhibition of nicotine-induced cancer-related inflammation. Real-time PCR analysis identified that exposure to nicotine rendered an increased $\mathrm{NF}-\kappa \mathrm{B}$ p65 and a reduced I $\mathrm{B}-\alpha$ mRNA levels in both A549 and H1299 cells; nicotine with baicalin, baicalein or wogonin treatment reverted $\mathrm{NF}-\kappa \mathrm{B}$ p65 level in both cell lines and only I $\mathrm{B}-\alpha$ level in H1299 cell lines (Fig. 9A and B). Western blot data indicated that although nicotine-exposed A549 cells did not induce apparent $\mathrm{NF}-\kappa \mathrm{B}$ p 65 or $\mathrm{I} \kappa \mathrm{B}-\alpha$ protein changes, treatment with nicotine along with baicalin, baicalein or wogonin downregulated protein expression of $\mathrm{NF}-\kappa \mathrm{B}$ p65 (Fig. 9C and D). In addition, as expected, NF- $\kappa \mathrm{B}$ p 65 and $\mathrm{I} \kappa \mathrm{B}-\alpha$ protein expression of each group in $\mathrm{H} 1299$ cells were consistent with their mRNA levels.

\section{Discussion}

Nicotine has been found to induce proliferation and metastasis of lung cancer cells. Recently, many anticancer natural substances, not generating side effects, have elicited considerable interests. This study furnishes the first evidence that flavonoid components in Scutellaria baicalensis could inhibit nicotine-induced proliferation, migration and lung cancerassociated inflammation.

The imbalance between proliferation and apoptosis leads to limitless cell proliferation that ultimately develops into a tumor, making induction of apoptosis the main target of current chemotherapeutic agents aimed at cancer prevention and treatment (30). Members of bcl-2 family are critical regulators of apoptosis; for example, bcl-2, the first discovered cell death regulator, promotes cell survival, and bax activates the effector pathways of apoptosis. Our study demonstrated that baicalin, baicalein and wogonin abrogates nicotine-induced lung cancer A549 and H1299 cell apoptosis. Mechanistically, we observed a ratio reduction in bcl-2/bax protein expression in A549 cells but not in H1299 cells (i.e., increase of the bax and decrease of bcl-2 levels). Therefore, other factors are probably involved in the anti-apoptotic process of flavones in H1299 cells. Caspases, especially caspase-3, plays a pivotal role in the final common pathway of apoptosis (31). In the present study, a significant increase in the mRNA expression of pro-caspase-3 was observed after nicotine treatment in both A549 and H1299 cells, while in protein expression, nicotine only upregulated pro-caspase-3 in H1299 cells, but not in A549 cells. This provides evidence that the responses of different cell lines to nicotine are diverse. Baicalin, baicalein and wogonin significantly inhibited pro-caspase-3 mRNA and protein expression triggered by nicotine in both cell types, showing the involvement of caspase- 3 in the flavones-induced apoptosis.

Metastasis is a vital characteristic of malignancy and primary cause of death for most cancer patients. Exposure of nicotine promotes the invasive and migratory ability of lung cancer cells and transplanted tumor in a mouse model $(13,14)$. In this study, we also found similar effect of nicotine on A549 and H1299 cells; in addition, our results demonstrated that all flavones in Scutellaria inhibited nicotine-induced invasion in both cell lines and migration in A549 cells, and only baicalein suppressed nicotine-induced migration in H1299 cells. To gain further knowledge of the mechanism of these flavones on depressing tumor metastasis, expression of MMP-2 and MMP-9 was detected by real-time PCR and western blotting. MMPs act as critical mediators of degrading and remodeling cell extracellular matrix so as to initiate metastasis (32). It has been reported that baicalein inhibits pulmonary carcinogenesis-associated inflammation by interfering with MMP-2 and MMP-9 expression (33). Our data showed that baicalein and wogonin downregulated the gene and protein expression of MMP-2 and MMP-9 in both A549 and H1299 cell lines, indicating a possible role for MMP-2 and MMP-9 in the process. Interestingly, baicalin only caused some of the gene or protein changes of MMP-2 or MMP-9 in cancer cells, and therefore alternative mechanisms may ccause the anti-metastatic effect of baicalin.

Inflammation plays a key role in lung cancer promotion and progression. A considerable portion of lung cancer patients also suffer from chronic obstructive pulmonary disease (COPD). Inflammatory mediators that are thought to contribute to the pathogenesis of COPD may also contribute to lung carcinogenesis (34). Takahashi et al reported that the inflammatory cells promote lung cancer cell proliferation to increase IL- 6 and TNF- $\alpha$ production by triggering IKK- $\beta$ / $\mathrm{NF}-\kappa \mathrm{B}$ pathway and subsequently these cytokines increase proliferation of alveolar epithelial cells (28). Thus, our study investigated TNF- $\alpha$ and IL-6 levels in cell culture supernatant. Except for wogonin on nicotine-induced IL-6 expression, all phytochemicals significantly inhibited TNF- $\alpha$ and IL-6 expression on nicotine-induced A549 and H1299 cells, which is in line with our previous finding that baicalin inhibits TNF- $\alpha$ and IL-6 levels in serum and bronchoalveolar lavage fluid of COPD mice (24). It is indicated that antitumor effect of these 
flavones is possibly related to the inhibition of lung cancerassociated inflammation. NF- $\kappa \mathrm{B}$ acts as a critical mechanistic link between inflammation and cancer. Its activation is able to upregulate the expression of tumor promoting cytokines, such as TNF- $\alpha$ or IL-6, and survival genes, such as bcl-2. We have reported that baicalin attenuates pulmonary inflammation by inhibiting NF- $\kappa \mathrm{B}$ activation in cigarette smoke-induced COPD rat model (24). In the present study, expression of NF- $\mathrm{NB}$ p65 induced by nicotine in A549 cells differed from that in H1299 cells, depending on their p53 status (35). Flavones in Scutellaria dramatically downregulated $\mathrm{NF}-\kappa \mathrm{B}$ p 65 protein expression in both cell types and elevated $\mathrm{I} \kappa \mathrm{B}-\alpha$ protein expression in $\mathrm{H} 1299$ cells. These findings suggest baicalin, baicalein and wogonin may play an anti-inflammatory microenvironment role during nicotine-induced malignant progression.

Our overall observation proves for the first time that flavonoid components in Scutellaria baicalensis inhibit nicotine-induced proliferation, migration and lung cancerassociated inflammation in vitro. These findings suggest that baicalin, baicalein and wogonin should be further explored as promising chemotherapeutic agents against nicotine-induced tumor growth and metastasis.

\section{Acknowledgements}

This study was funded by grants from National Basic Science Program of China (2009CB523000) and National Natural Science Foundation of China (81102541).

\section{References}

1. Jemal A, Bray F, Center MM, Ferlay J, Ward E and Forman D: Global cancer statistics. CA Cancer J Clin 61: 69-90, 2011.

2. Chen W, Zhang S and Zou X: Estimation and projection of lung cancer incidence and mortality in China. Zhongguo Fei Ai Za Zhi 13: 488-493, 2010 (In Chinese).

3 . Hecht SS: Cigarette smoking and lung cancer: chemical mechanisms and approaches to prevention. Lancet Oncol 3: 461-469, 2002.

4. Zhang J, Ou JX and Bai CX: Tobacco smoking in China: prevalence, disease burden, challenges and future strategies Respirology 16: 1165-1172, 2011.

5. Egleton RD, Brown KC and Dasgupta P: Nicotinic acetylcholine receptors in cancer: multiple roles in proliferation and inhibition of apoptosis. Trends Pharmacol Sci 29: 151-158, 2008.

6 . Thunnissen FB: Acetylcholine receptor pathway and lung cancer. J Thorac Oncol 4: 943-946, 2009.

7. Maneckjee R and Minna JD: Opioid and nicotine receptors affect growth regulation of human lung cancer cell lines. Proc Natl Acad Sci USA 87: 3294-3298, 1990

8. Tournier JM and Birembaut P: Nicotinic acetylcholine receptors and predisposition to lung cancer. Curr Opin Oncol 23: 83-87, 2011.

9. Lam DC, Girard L, Ramirez R, et al: Expression of nicotinic acetylcholine receptor subunit genes in non-small-cell lung cancer reveals differences between smokers and nonsmokers. Cancer Res 67: 4638-4647, 2007.

10. Kelly PN and Strasser A: The role of Bcl-2 and its pro-survival relatives in tumourigenesis and cancer therapy. Cell Death Differ 18: 1414-1424, 2011.

11. Leber B, Geng F, Kale J and Andrews DW: Drugs targeting Bcl-2 family members as an emerging strategy in cancer. Expert Rev Mol Med 12: e28, 2010.

12. Xin M and Deng X: Nicotine inactivation of the proapoptotic function of Bax through phosphorylation. J Biol Chem 280: 10781-10789, 2005.

13. Dasgupta P, Rizwani W, Pillai S, et al: Nicotine induces cell proliferation, invasion and epithelial-mesenchymal transition in a variety of human cancer cell lines. Int J Cancer 124: 36-45, 2009.
14. Davis R, Rizwani W, Banerjee S, et al: Nicotine promotes tumor growth and metastasis in mouse models of lung cancer. PLoS One 4: e7524, 2009.

15. Nelson AR, Fingleton B, Rothenberg ML and Matrisian LM: Matrix metalloproteinases: biologic activity and clinical implications. J Clin Oncol 18: 1135-1149, 2000.

16. Bayramoglu A, Gunes HV, Metintas M, Degirmenci I, Mutlu F and Alatas F: The association of MMP-9 enzyme activity, MMP-9 C1562T polymorphism, and MMP-2 and -9 and TIMP-1, $-2,-3$, and -4 gene expression in lung cancer. Genet Test Mol Biomarkers 13: 671-678, 2009.

17. Herbst RS, Yano S, Kuniyasu H, et al: Differential expression of E-cadherin and type IV collagenase genes predicts outcome in patients with stage I non-small cell lung carcinoma. Clin Cancer Res 6: 790-797, 2000.

18. Klein G, Vellenga E, Fraaije MW, Kamps WA and de Bont ES: The possible role of matrix metalloproteinase (MMP)-2 and MMP-9 in cancer, e.g. acute leukemia. Crit Rev Oncol Hematol 50: 87-100, 2004

19. Zong Y, Zhang ST and Zhu ST: Nicotine enhances migration and invasion of human esophageal squamous carcinoma cells which is inhibited by nimesulide. World J Gastroenterol 15: 2500-2505, 2009.

20. Lee HZ, Leung HW, Lai MY and Wu CH: Baicalein induced cell cycle arrest and apoptosis in human lung squamous carcinoma CH27 cells. Anticancer Res 25: 959-964, 2005.

21. Leung HW, Yang WH, Lai MY, Lin CJ and Lee HZ: Inhibition of 12-lipoxygenase during baicalein-induced human lung nonsmall carcinoma H460 cell apoptosis. Food Chem Toxicol 45: 403-411, 2007.

22. Du G, Han G, Zhang S, et al: Baicalin suppresses lung carcinoma and lung metastasis by SOD mimic and HIF-1alpha inhibition. Eur J Pharmacol 630: 121-130, 2010.

23. Li-Weber M: New therapeutic aspects of flavones: the anticancer properties of Scutellaria and its main active constituents Wogonin, Baicalein and Baicalin. Cancer Treat Rev 35: 57-68, 2009.

24. Lixuan Z, Jingcheng D, Wenqin Y, Jianhua H, Baojun L and Xiaotao F: Baicalin attenuates inflammation by inhibiting NF-kappaB activation in cigarette smoke induced inflammatory models. Pulm Pharmacol Ther 23: 411-419, 2010.

25. $\mathrm{Li} \mathrm{L}, \mathrm{Bao} \mathrm{H}, \mathrm{Wu}$ J, et al: Baicalin is anti-inflammatory in cigarette smoke-induced inflammatory models in vivo and in vitro: a possible role for HDAC2 activity. Int Immunopharmacol 13: 15-22, 2012.

26. Petty TL: Are COPD and lung cancer two manifestations of the same disease? Chest 128: 1895-1897, 2005.

27. Yang IA, Relan V, Wright CM, et al: Common pathogenic mechanisms and pathways in the development of COPD and lung cancer. Expert Opin Ther Targets 15: 439-456, 2011.

28. Takahashi H, Ogata H, Nishigaki R, Broide DH and Karin M: Tobacco smoke promotes lung tumorigenesis by triggering IKKbeta- and JNK1-dependent inflammation. Cancer Cell 17: 89-97, 2010.

29. Livak KJ and Schmittgen TD: Analysis of relative gene expression data using real-time quantitative PCR and the 2(-Delta Delta C(T)) method. Methods 25: 402-408, 2001.

30. Karunagaran D, Joseph J and Kumar TR: Cell growth regulation. Adv Exp Med Biol 595: 245-268, 2007.

31. Fennell DA: Caspase regulation in non-small cell lung cancer and its potential for therapeutic exploitation. Clin Cancer Res 11: 2097-2105, 2005.

32. Steeg PS: Tumor metastasis: mechanistic insights and clinical challenges. Nat Med 12: 895-904, 2006.

33. Chandrashekar N, Selvamani A, Subramanian R, Pandi A and Thiruvengadam D: Baicalein inhibits pulmonary carcinogenesisassociated inflammation and interferes with COX-2, MMP-2 and MMP-9 expressions in-vivo. Toxicol Appl Pharmacol 261: 10-21, 2012.

34. Tauler $\mathrm{J}$ and Mulshine JL: Lung cancer and inflammation: interaction of chemokines and hnRNPs. Curr Opin Pharmacol 9: 384-388, 2009.

35. Puliyappadamba VT, Cheriyan VT, Thulasidasan AK, et al: Nicotine-induced survival signaling in lung cancer cells is dependent on their p53 status while its downregulation by curcumin is independent. Mol Cancer 9: 220, 2010. 